\title{
Original
}

\section{Construction of a Novel Single Double-Conditional shRNA Expression Vector}

\author{
Tomoyuki Matsuoka, Hideto Oyamada, Masahideo NaKano, \\ Masayuki SomeI, Eiji Tomoyori, Yasuyuki Kondo \\ and Katsuji OguchI
}

\begin{abstract}
Silencing of gene expression by RNA interference (RNAi) has become a widely used tool for assessing gene function in a fast and easy manner. An important advance in the RNAi field was the discovery that plasmid-based short hairpin RNA transcription can substitute for synthetic small interference RNAs both in vitro and in vivo. The constitutive knockdown of gene expression by RNAi can limit the scope of experiments, especially if the inhibition of genes leads to cell lethality, which prevents in vivo functional analysis. We have generated double-conditional short hairpin RNA from a single vector that can regulate its own transcription by a combination of tetracycline-inducible and Cre-loxP systems. This vector will be useful for the control of short hairpin RNA expression in a spatially-regulated manner by $\mathrm{Cre}$ recombinase as well as a temporally-regulated manner by tetracycline induction. These control features provide for the specific silencing of genes to facilitate functional genetic analysis in mammals in vivo.
\end{abstract}

Key words : RNA interference, short hairpin RNA, double-conditional vector, tetracycline-inducible system, Cre-LoxP system

\section{Introduction}

RNA interference (RNAi) is a posttranscriptional gene silencing phenomenon that is induced by double-stranded RNA (dsRNA) and was first identified in the nematode, Caenorhabditis elegans ${ }^{1}$. A member of RNase III enzyme family, Dicer, cleaves long dsRNAs introduced into cells during the initial steps of RNAi into small interfering RNAs (siRNAs) ${ }^{2)}$. These siRNA duplexes are unwound by an RNA helicase and are incorporated into the multi-protein RNA-induced silencing complex (RISC). Annealing of the siRNA to homologous mRNA results in mRNA degradation and suppression of the target gene expression ${ }^{3)}$. This phenomenon is conserved in evolutionarily diverse organisms, but gene silencing by RNAi is difficult to detect in mammalian systems because long dsRNAs result in activation of antiviral pathways, nonspecific inhibition of translation and cytotoxic responses ${ }^{4)}$. The use of synthetic $21 \sim 25$ bp siRNA to down-modulate target genes is one way to circumvent the cytotoxic dsRNA-activated pathways in mammals ${ }^{5)}$. Chemically synthesized siRNAs introduced into mammalian cells by transient transfection have limited 
efficacy in gene silencing. The RNA-dependent RNA polymerase protein (RdRP), that forms the siRNA-replication systems in fungi, plants and nematodes, is not found in mammals ${ }^{6}$. To overcome this limitation, several mammalian expression vectors have been designed to direct the constitutive synthesis of short hairpin RNAs (shRNA) as precursors of siRNA-like transcripts ${ }^{7}$. Most of these vectors have used polymerase III (polIII) promoters including the U6, H1, and tRNA promoters, which direct the synthesis of small, noncoding transcripts with 3'-ends defined by termination within a stretch of four to five thymidine residues. This has the advantage of generating higher levels of siRNAs than polymerase II (pol II) promoters ${ }^{8)}$.

A major limitation of polIII promoters is that they do not control silencing in a time- and/or tissue- specific manner because they are constitutively and ubiquitously active, which has limited the scope of experiments, especially if gene silencing results in lethality. Recently developed conditional expression vectors enable the controlled production of shRNAs. These vectors permit similar analytical refinement to conditional gene knockout mice, which utilize either the Cre-loxP recombination system ${ }^{9)}$ or the tetracycline (Tet) -regulated system ${ }^{10)}$. We have generated novel conditional shRNA vectors that can regulate its silencing in a double conditional manner by the combination of both systems. These vectors will allow spatial and temporal control of shRNA expression in vivo and should facilitate functional genetic analysis in mammals.

\section{Materials and Methods}

\section{Generation of plasmids}

To generate a double conditional single shRNA plasmid vector, the Cre-loxP conditional system was added to a plasmid containing the Tet-controlled gene silencing system. A plasmid containing the first modified loxP site [hereafter termed "TATA-lox"11)], followed by the terminator polyA $(+)$ and a SmaI restriction site, was generated using self-ligated PCR-products from the pSingle-tTs-shRNA plasmid vector [Clontech, Palo Alto, CA, USA ${ }^{12)}$ ] as the template using the following oligonucleotides; Sma I + Xho I-F, 5'-CCGGGAACGCGCGGTGACCCTCGA GGGACAAGCTTATCTATGTCG-3', TATALOX1-R, 5'-GAAAAAAACCAAGGCTTATAACTTC GTATAATTTATACTATACGAAGTTATAATTACTTTCAAGTTACGGTAAGCATATG-3’

The resulting plasmid was digested with SmaI and XhoI and ligated to the following annealed oligonucleotides; TATALOX2-F, 5'-GGGAGAGCCTGCAGGATAACTTCGTATAGTAT AAATTATACGAAGTTATAAGC-3'; TATALOX2-R, 5'-TCGAGCTTATAACTTCGTATAATTT ATACTATACGAAGTTATCCTGCAGGCTCTCCC-3' in order to inset the second TATAlox site preceded by an Sbf I restriction site.

The resulting construct was then digested with Sma I plus Sbf I, and ligated to a DNA cassette containing a Sma I restriction site followed by the beta-actin promoter and the SNAPtag-rabbit beta-globin polyadenylation signal followed by an Nsi I restriction site to finally generate the pSingle-tTs-2lox shRNA vector.

\section{Cre-mediated DNA recombination}

An aliquot of $10 \mathrm{ng}$ of pSingle-tTs-2lox shRNA vector was incubated for $16 \mathrm{~h}$ with Cre 
recombinase (New England Biolabs, Inc., Ipswich, MA, USA), according to manufacturer's instructions, in order to excise the stuffer sequence between the two TATAlox sites. The digested vector was transformed into Ecos competent cells of E. coli DH5 $\alpha$ (Nippon Gene Co., Ltd., Tokyo, Japan) and was analyzed by restriction enzyme digestion and DNA sequencing to confirm the sequence of the novel shRNA expression vector. The DNA sequencing reactions were performed in a BioRad DNA Engine Dyad PTC-220 Peltier Thermal Cycler (Bio-Rad Laboratories, Inc., Hercules, CA, USA) using a ABI BigDye ${ }^{\mathrm{TM}}$ Terminator v3.1 Cycle Sequencing Kit with AmpliTaq DNA polymerase (Applied Biosystems, Inc., Foster City, CA, USA), following the protocols supplied by the manufacturer. The sequencing reactions were then subjected to electrophoresis in an ABI 3730xl sequencer (Applied Biosystems, Inc.).

\section{Nucleotide sequence analysis}

The genetic information software "GENETYX" (version 6, Genetyx Cor., Osaka, Japan) was used to design oligonucleotides and for nucleotide sequences analyses.

\section{Cell culture and DNA transfection}

Human embryonic kidney 293 (HEK293) cells were maintained in Dulbecco's modified Eagle medium (Wako Pure Chemicals Industries, Ltd., Osaka, Japan) with $10 \%$ fetal bovine serum (HyClone Lab. Inc., South Logan, UT, USA), and $100 \mathrm{units} / \mathrm{mL}$ penicillin, $100 \mu \mathrm{g} / \mathrm{mL}$ streptomycin antibiotics (Wako Pure Chemicals Industries). HEK293 cells were co-transfected with the shRNA expression vectors containing the shRNA oligonucleotide 5'-TCGAGGCAAG CTGACCCTGAAGTTCTTCAAGAGAGAACTTCAGGGTCAGCTTGCTTTTTTACGCGTA-3' for destabilized 2 enhanced green fluorescent protein ( $\mathrm{d} 2 \mathrm{EGFP}$, antisense nucleotide sequence is underlined ${ }^{13)}$ and the d2EGFP expression vector (Clontech) ${ }^{14)}$. The cells were plated on polyd-lysine-coated glass-bottomed 35- mm culture dishes (MatTek, Ashland, MA, USA) and were transfected by the calcium phosphate precipitation method ${ }^{15)}$ using a Profection Mammalian Transfection System Kit (Promega, Madison, WI, USA). Transfected cells were washed $4 \mathrm{~h}$ after the transfection and allowed to grow in fresh medium for $24 \mathrm{~h}$. The medium was then replaced with the same growth medium either containing $1 \mu \mathrm{g} / \mathrm{mL}$ doxycycline (Dox; Sigma-Aldrich Co., St. Louis, MO, USA) to induce the production of shRNA or without Dox as the non-induced control experiments.

\section{Microscopic detection of d2EGFP fluorescence}

The Dox-induced inhibition of the d2EGFP expression in HEK293 cells was assayed at $48 \mathrm{~h}$ after application of Dox. The cells were washed twice with phosphate-buffered saline (PBS) and fixed with 4\% formaldehyde in PBS for 15 in at room temperature. All transfected HEK293

cells were viewed under an inverted fluorescent microscope Eclipse-Ti-U (Nikon, Tokyo, Japan) equipped with a cooled charge-coupled device camera DS-2MBWc (Nikon). 


\section{Results}

\section{Modified-loxP insertion into Tet-responsive hybrid U6 promoter}

The commonly used polIII promoter-based shRNA vectors are ubiquitously active and do not allow control of tissue-specific gene silencing. To enable the controlled production of shRNA specifically in a tissue of interest, conditional expression vectors were developed that offer a similar analytical refinement as the conditional knockout mice with the Cre-loxP recombination technologies. Our aim was to modify the commercially-available pSingle-tTs-shRNA vector to control shRNA expression in a Cre-dependent manner.

The U6 promoter is similar to other polIII promoters with a compact organization consisting of a tightly spaced TATA box, a proximal sequence element (PSE) and a distal sequence element (DSE). The PSE and the TATA box are absolutely necessary while the DSE is partially dispensable for transcriptional activity ${ }^{11)}$. In fact, the pSingle-tTs-shRNA commercially available vector has the PSE and the TATA box in its modified Tet-responsive polIII hybrid promoter (PTREmodU6) but does not have a DSE. Sequence alignments of the human U6 promoter with Tet-responsive modified U6 promoters are shown in Fig. 1. The normal spacing between the PSE and the 17-bp TATA box as well as between the TATA box and the 25-bp transcription start site must be preserved, because even small changes in the length of this region markedly impair promoter activity ${ }^{16)}$. This consideration precludes the incorporation of an additional classic loxP sequence because the 34-bp loxP site that remains after Cre-mediated recombination would increase the spacing between the TATA box and both the PSE and the transcription start site.

To overcome these limitations, we utilized a bifunctional loxP site, termed the "TATA-lox" site $^{11)}$, which contains a functional TATA box in its spacer region. The insertion of the TATAlox site replaced the TATA box in the PTREmodU6 promoter, and the spacing between the TATA box and both the PSE and the transcription start site is not altered (Fig. 2). Cremediated recombination of this modified vector containing two TATA-lox sites in the same orientation [“TATA-lox"-“terminator (TTTTTT)"-“stuffer sequence”-“TATA-lox", termed "TATA-2lox"] resulted in the loss of one PvuII site in the stuffer sequence to yield a recombined sequence, termed "TATA-1lox"(Fig. 3). DNA sequence analysis confirmed that the spacing between the TATA box and PSE was $17 \mathrm{bp}$ and that between the TATA box and the transcription start site was $25 \mathrm{bp}$ after Cre-mediated recombination (Fig. 4).

To evaluate whether these modified shRNA vectors could functionally regulate conditional gene-silencing abilities, the d2EGFP reporter expression vector was co-transfected into HEK293 cells with the shRNA of vectors (TATA-2lox, TATA-1lox or the original pSingle-tTs-shRNA vector) containing the shRNA sequence corresponding to the coding region of EGFP. Three days after transfection, a strong green fluorescence signal was observed in many cells, which demonstrated that d2EGFP was efficiently expressed under the control of the CMV promoter. The d2EGFP fluorescent intensity in the cytosol was not decreased in the absence of Dox, in all three combinations of co-transfections [Fig. 5 (A), (B) and (C), right panels]. The application 


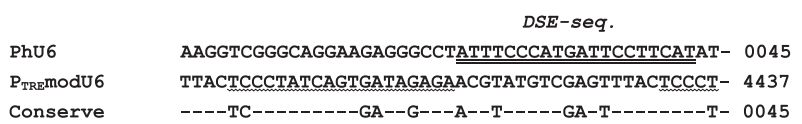

TTGCATATACGATACAAGGCTGTTAGAGAGATAATTAGAATTAATTTGACTGTAAACAC- 0104 ATCAGTGATAGAGAACGTATGTCGAGTTTACTCCCTATCAGTGATAGAGAACTATGTC- 4495

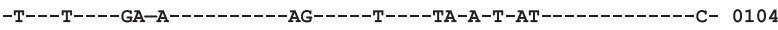

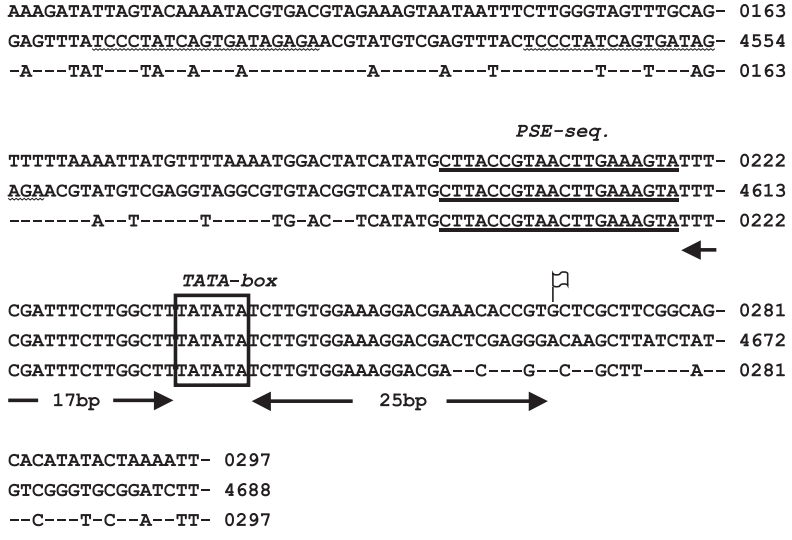

Fig. 1. Sequence comparison of human U6 and tetracyclineresponsive modified U6 promoters.

Sequence alignment analysis shows that the proximal sequence element (PSE; bold underlined) and the TATA box (open-boxed) are strictly conserved between the two promoters. The distal DSE (double underlined) in the wild-type human U6 promoter "PhU6" (first lane) is lost and partially replaced with one of tet $\mathrm{O}_{2}$ sequences (wavy underlined) of the modified promoter "PTREmodU6" (second lane) in the pSingle-tTs-shRNA vector. The transcription start site is indicated $(P)$ and conserved nucleotides between the two promoters are shown in the third lane.

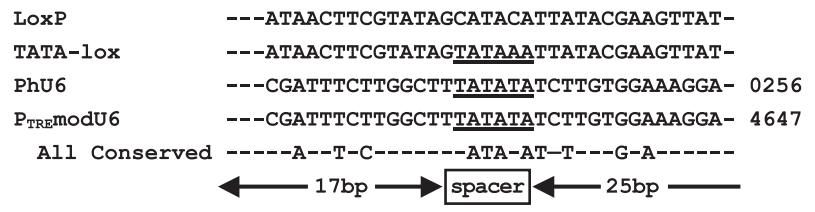

Fig. 2. Sequence comparison of LoxP, TATA-lox and U6 promoters. The sequences of a LoxP site (1st lane), a TATA-lox site (2nd lane), the PhU6 promoter (3rd lane) and the PtREmodU6 promoter (4th lane) are aligned. The lowest lane indicates the conserved nucleotide among all four sequences, LoxP, TATA-lox, PhU6 and PtremodU6.

of Dox induced a significant decrease in the d2EGFP intensity of the co-transfected cells with Cre-recombinase-treated shRNA pSingle-tTs-TATA-1lox-shRNA [Fig. 5 (B), left panels]. The suppressive action of this modified vector was comparable to the original pSingle-tTs-shRNA vector [Fig. 5 (B) and (C), left panels]. No inhibitory effect was observed in the co-transfected cells with pSingle-tTs-TATA-2lox-shRNA in the presence of Dox [Fig. 5 (C), left panels]. 
(A)

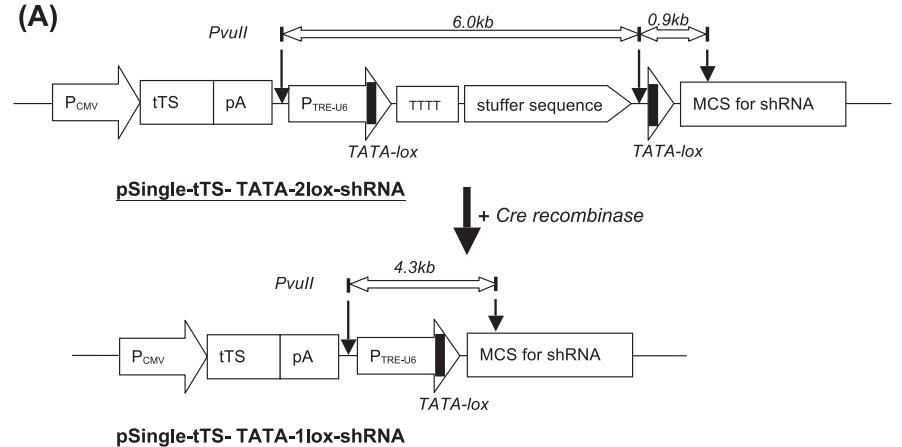

(B)

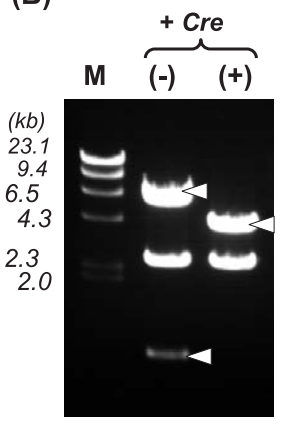

Fig. 3. Cre-mediated recombination of double-conditional shRNA vector.

(A) A schematic representation of the novel shRNA expression constructs before and after Cre-mediated recombination is shown. Vertical small arrows indicate Pvu II restriction sites in each vector. The expected Pvu II restriction fragment sizes are indicated before and after the Cre-mediated recombination. The resulting Pvu II fragments are produced by the excision of a transcription termination signal (designated as "TTTT") and a stuffer sequence between the two TATA-lox sites in a "pSingle-tTs-TATA-2lox-shRNA" vector. The cytomegalovirus promoter, a transcriptional trans-silencer and a polyadenylation signal are indicated by PCMv, tTs and pA, respectively.

(B) Agarose gel electrophoretic analysis was used to verify the Cre-mediated recombination. The (-) and $(+)$ symbols indicate the + Cre samples before and after Cre recombinase treatment. Open arrowheads indicate the fragments shown in (A). The $\lambda$ /HindIII DNA size marker is indicated by $(\mathrm{M})$.

\section{(A) pSingle-tTS-TATA-2lox-shRNA}
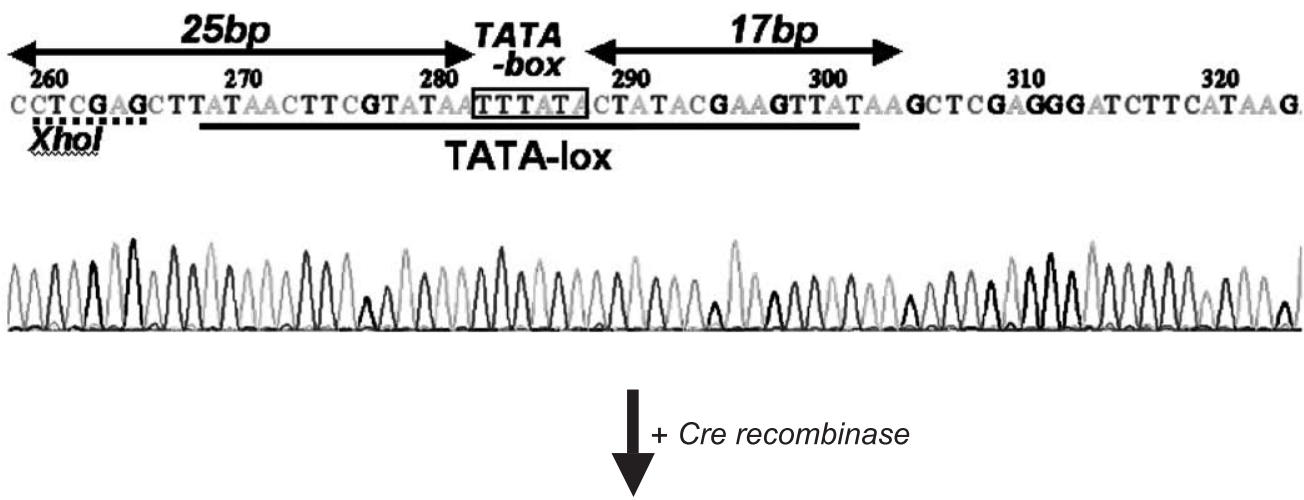

(B) pSingle-tTS- TATA-1lox-shRNA
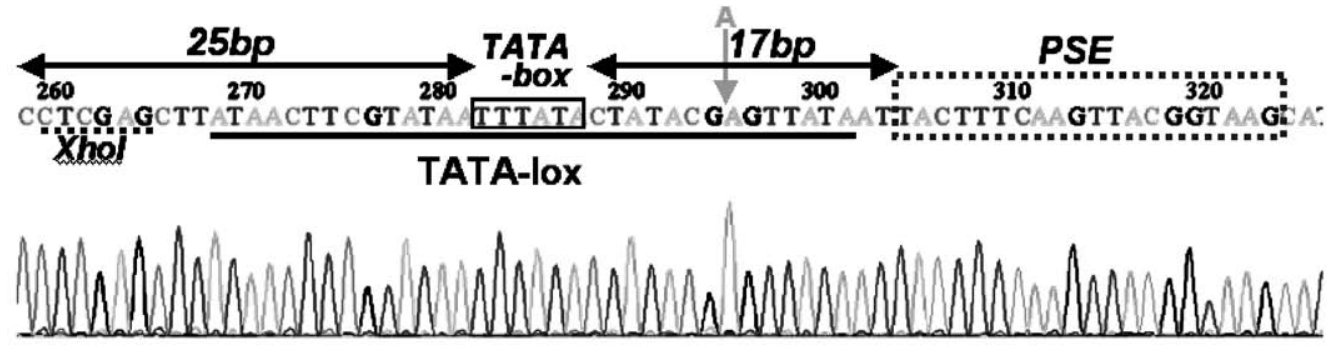

Fig. 4. Sequence confirmation of Cre-mediated recombination in the double conditional shRNA vector.

Each chart record shows the complementary nucleotide sequences from the samples before (A) or after (B) Cre-recombinase treatment. The PSE sequence occurs $17 \mathrm{bp}$ downstream of the TATA box in (B) but not in (A). The doubling peak value for the adenine (A) signal is indicated by the green arrow and represents the sum of two consecutive A signals. 


\section{(A) pSingle-tTS- TATA-2lox-shRNA for d2EGFP}

$(+)$ Dox

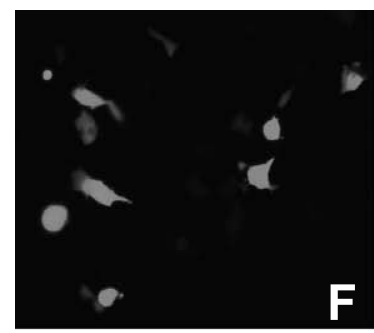

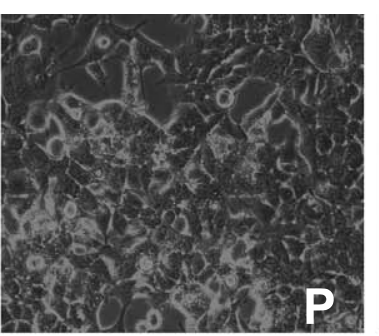

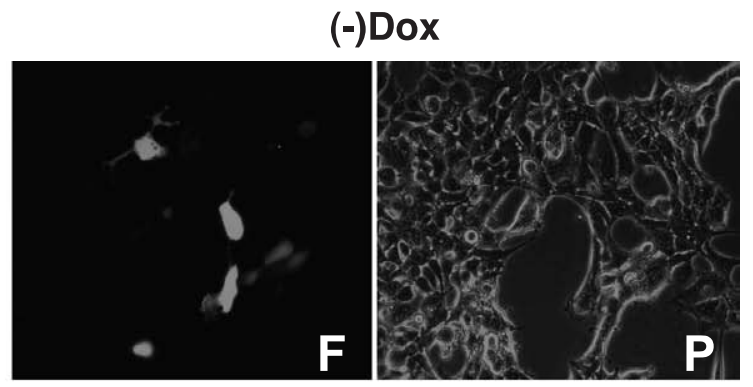

(B) pSingle-tTS- TATA-1lox-shRNA for d2EGFP

(+) Dox

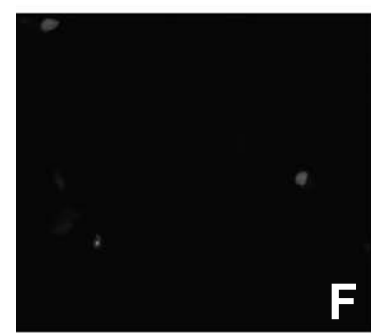

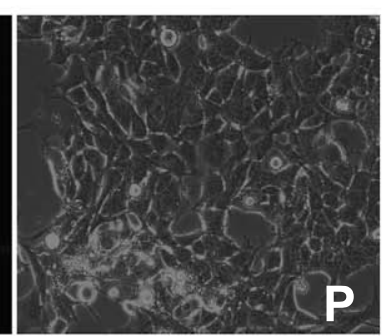

(-) Dox

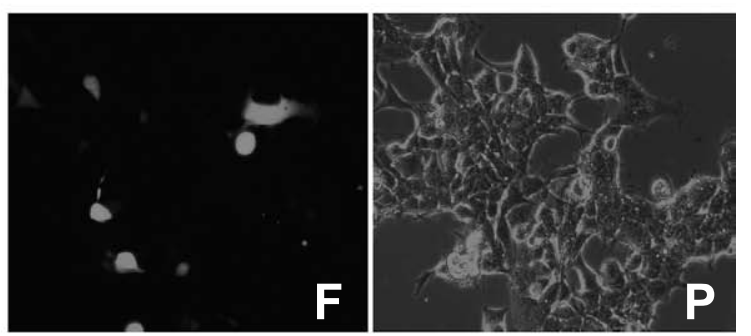

(C) pSingle-tTS-shRNA for d2EGFP

(+) Dox

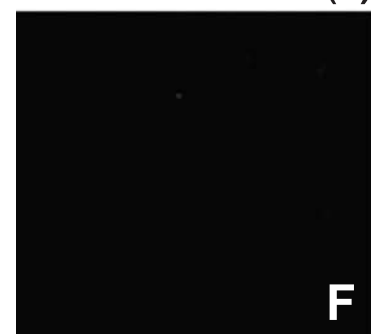

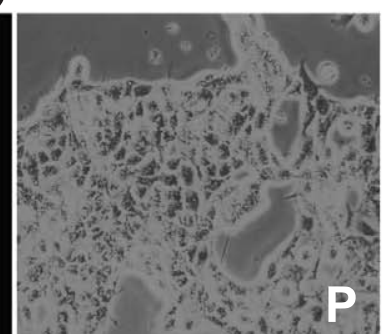

(-) Dox

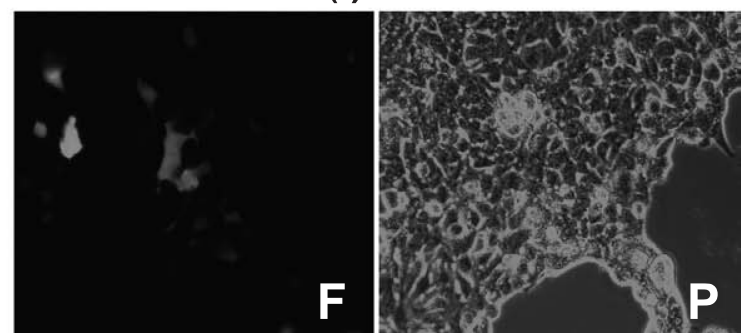

Fig. 5. Doxycycline-inducible knockdown of d2EGFP expression in HEK293 cells by double conditional shRNA vectors.

Fluorescence images $(\mathrm{F})$ were taken from cells transfected with the d2EGFP expression vector and co-transfected with the pSingle -tTs-TATA-2lox-shRNA vector (A), pSingle -tTs-TATA-1lox-shRNA vector (B) or the original pSingle -tTs-shRNA vector (C). Identical exposure times were used for capturing all fluorescence images. The corresponding phase-contrast images $(\mathrm{P})$ of the same fields of culture dishes were also captured. In the absence of doxycycline $[(-)$ Dox $]$, d2EGFP fluorescence could be detected consistently in the cytosol of many cells $(\mathrm{A}),(\mathrm{B})$ and $(\mathrm{C})$. Application of Dox $[(+)$ Dox] into the culture medium induced the silencing of d2EGFP expression in the co-transfected cells as shown in (B) and (C) but not in (A).

\section{Discussion}

Since the development of gene targeting technologies, the gold standard for the analysis of gene function in mammals has been the generation of gene knockout $(\mathrm{KO})$ mice $^{17)}$. The production of loss-of-function alleles in the mouse remains time consuming and costly despite significant technological improvements. Recently, the constitutive expression of shRNAs driven 
by polIII promoters has been demonstrated to functionally silence gene expression in transgenic mice. This suggests that RNAi-based technologies might represent a convenient alternative to gene targeting through homologous recombination ${ }^{18)}$. Current approaches for the use of transgenic RNAi have a major limitation because they result in constitutive gene silencing in all tissues and do not allow regulated expression of shRNA. Improvements in this technology have permitted refined analyses of gene function in specific tissues, based on conditional $\mathrm{KO}$ strategies including Cre-loxP-mediated recombination. Transgenic mice produced by this technique can result in early embryonic lethality by the inhibition of gene expression, whereas others show no abnormalities in their general phenotype. This results from compensatory gene induction of one or more genes that act as counterparts to the targeted gene and maintain whole-body homeostasis during growth and/or development. In both cases, an analysis of the role(s) of genes-of-interest in adult transgenic mice is problematic.

To overcome these difficulties, we have combined a drug-inducible shRNA expression system with the Cre-loxP regulated system ${ }^{9,17)}$. In a conventional drug-inducible expression system, such as the Tet inducible system ${ }^{10)}$, two kinds of genetic regulatory elements in two distinct vectors must be introduced into an individual cell or body. One is the Tet-responsive element (TRE) containing minimal CMV promoter, and the other is the gene of Tet repressor (TetR) gene. The TetR protein blocks transcription by binding to the Tet operator sequences (tetO) of the TRE in the absence of Tet or its derivatives.

The novel vector described here was developed from the commercially available pSingle-tTsshRNA plasmid (Clontech) ${ }^{12}$. This novel vector has two essential components combined in a single plasmid : 1) CMV promoter/enhancer-controlled expression of the Tet regulatory protein, a transcriptional trans-silencer tTs, and 2) a Tet-inducible shRNA expression cassette including the modified Tet-responsive polIII hybrid promoter (PTREmodU6). Therefore, only a single round of gene transfer into the host cells or fertilized eggs is required for drug-inducible gene silencing.

In conclusion, we have constructed a novel single shRNA expression vector for gene silencing that can be double-conditioned by the Tet-inducible system and the Cre-loxP system. The Tet control system can control the onset and termination of genetic manipulations by application of Tet or its derivatives to either cells or animals. The Cre-loxP system can regulate the site of Cre recombinase expression in a tissue-specific manner in transgenic animals by utilizing a tissue-specific promoter. More recently, we have replaced the CMV promoter/enhancer with the human elongation factor 1 alpha $(\mathrm{EF} 1 \alpha)$ promoter that allows expression of TetR in a variety of mammalian cells, including hematopoietic or stem cells, without the transgene silencing associated with CMV promoter ${ }^{19-21)}$. Transgenic mice with this double conditional shRNA expression vector will be crossed with the genetically engineered mice expressing the Cre- recombinase in a tissue-specific manner to obtain the mice that can express the shRNA for silencing the gene of interest in both spatial (tissue-specific) and temporal (Tet-inducible) manners. We anticipate that genetically engineered mice with this double conditional shRNA expression system will be exceptionally useful for investigating the functional roles of gene of interest in vivo. 


\section{References}

1) Fire $\mathrm{A}, \mathrm{Xu} \mathrm{S}$, Montgomery $\mathrm{MK}$, et al. Potent and specific genetic interference by double-stranded RNA in Caenorhabditis elegans. Nature. 1998;391:806-811.

2) Bernstein E, Caudy AA, Hammond SM, et al. Role for a bidentate ribonuclease in the initiation step of RNA interference. Nature. 2001;409:363-366.

3) Schwarz DS, Hutvangner G, Du T, et al. Asymmetry in the assembly of the RNAi enzyme complex. Cell. 2003;115:199-208.

4) Stark GR, Kerr IM, Williams BR, et al. How cells respond to interferons. Ann Rev Biochem. 1998;67:227-264.

5) Elbashir SM, Harborth J, Lendeckel W, et al. Duplexes of 21-nucleotide RNAs mediate RNA interference in cultured mammalian cells. Nature. 2001;411:494-498.

6) Zamore PD. Ancient pathways programmed by small RNAs. Science. 2002;296:1265-1269.

7) Brummelkamp TR, Bernards R, Agami R. A system for stable expression of short interfering RNAs in mammalian cells. Science. 2002;296:550-553.

8) Kato Y, Taira K. Expression of siRNA from a single transcript that induces multiple ribozymes in mammalian cells. Oligonucleotides. 2003;13:335-343.

9) Orban PC, Chui D, Marth JD. Tissue- and site-specific DNA recombination in transgenic mice. Proc Natl Acad Sci USA. 1992;89:6861-6865.

10) Gossen M, Bujard H. Tight control of gene expression in mammalian cells by tetracycline-responsive promoters. Proc Natl Acad Sci USA. 1992;89:5547-5551.

11) Ventura A, Meissner A, Dillon CP, et al. Cre-lox-regulated conditional RNA interference from transgenes. Proc Natl Acad Sci USA. 2004;101:10380-10385.

12) Kappel S, Matthess Y, Kaufmann M, et al. Silencing of mammalian genes by tetracycline-inducible shRNA expression. Nature Protoc. 2007;2:3257-3269.

13) Yoon SY, Choi JE, Hwang O, et al. Construction of a vector generating both siRNA and a fluorescent reporter: a siRNA study in cultured neurons. Mol Cells. 2004;18:127-130.

14) Li $\mathrm{X}$, Zhao $\mathrm{X}$, Fang $\mathrm{Y}$, et al. Generation of destabilized green fluorescent protein as a transcription reporter. $J$ Biol Chem. 1998;273:34970-34975.

15) Chen C, Okayama H. High-efficiency transformation of mammalian cells by plasmid DNA. Mol Cell Biol. 1987; 7:2745-2752.

16) Paule MR, White RJ. Survey and summary: transcription by RNA polymerases I and III. Nucleic Acids Res. 2000; 28:1283-1298.

17) Garcia-Otin AL, Guillou F. Mammalian genome targeting using site-specific recombinases. Front Biosci. 2006;11:1108-1136.

18) Kuhn R, Streif S, Wurst W. RNA interference in mice. Handb Exp Pharmacol. 2007;178:149-176.

19) Gopalkrishnan RV, Christiansen KA, Goldstein NI, et al. Use of the human EF-1alpha promoter for expression can significantly increase success in establishing stable cell lines with consistent expression: a study using the tetracycline-inducible system in human cancer cells. Nucleic Acids Res. 1999;27:4775-4782.

20) Hong S, Hwang DY, Yoon S, et al. Functional analysis of various promoters in lentiviral vectors at different stages of in vitro differentiation of mouse embryonic stem cells. Mol Ther. 2007;15:1630-1639.

21) Liu J, Jones KL, Sumer H, et al. Stable transgene expression in human embryonic stem cells after simple chemical transfection. Mol Reprod Dev. 2009;76:580-586.

[Received December 12, 2012 : Accepted December 21, 2012] 